\title{
RICHIESTE E ATTENUAZIONE: UN CONFRONTO TRA ITALIANO E PORTOGHESE BRASILIANO
}

\author{
REQUESTS AND MITIGATION: A COMPARISON BETWEEN ITALIAN AND BRAZILIAN \\ PORTUGUESE
}

\section{Elisabetta SANTORO}

Universidade de São Paulo

Abstract:

Lo studio, inserito nell'ambito della pragmatica cross-culturale, si concentra sull'atto linguistico della richiesta ed è stato realizzato sulla base di dati raccolti tramite un DCT (Discourse Completion Test) scritto, inviato elettronicamente a parlanti nativi di italiano e di portoghese brasiliano nella loro rispettiva lingua materna. Agli informatori è stato chiesto di scrivere che cosa avrebbero detto nelle situazioni descritte, le cui principali variabili erano il grado di imposizione e la distanza sociale tra gli interlocutori. L'obiettivo della ricerca, che grazie allo strumento utilizzato permette di raccogliere un elevato numero di dati ed è ampliabile e replicabile, è quello di analizzare contrastivamente i dati raccolti nelle due lingue, identificando la presenza di attenuatori all'interno della richiesta e l'effetto delle variabili selezionate, al fine di osservare analogie e differenze e, soprattutto, ricorrenze che possano rappresentare punti di riferimento anche per ricerche future. In questo articolo verranno presentati i primi risultati sulla base di un campione composto da 30 parlanti nativi di italiano e 30 di portoghese brasiliano.

PARole ChIAVE: richieste, attenuazione, pragmatica cross-culturale, italiano, portoghese brasiliano
Abstract:

In the perspective of Cross-Cultural Pragmatics, the study focuses on the speech act of the request and is based on data collected through a written DCT (Discourse Completion Test), sent electronically to native speakers of Italian and Brazilian Portuguese in their respective mother tongue. Informants were asked to write what they would have said in the described situations. The main variables were rank of imposition and social distance between the interlocutors. The aim of the research, which thanks to the instrument used allows to collect a large number of data and is expandable and replicable, is to analyze contrastively the data collected in the two languages, identifying the presence of modifiers in the request and the effect of the chosen variables, in order to observe similarities and differences, and above all, occurrences that may represent also a reference point for future research. This paper will present the first results based on a sample of 30 native speakers of Italian and 30 of Brazilian Portuguese.

KeYwords: Requests, Mitigation, CrossCultural Pragmatics, Italian, Brazilian Portuguese 


\section{normas}

\section{$1 \quad$ INTRODUZIONE}

Quello della variabilità è uno dei concetti chiave su cui si fonda la pragmatica linguistica che studia gli effetti del contesto su ciò che facciamo parlando e si occupa della lingua dal punto di vista degli utenti. Questo principio è centrale per la pragmatica cross-culturale o contrastiva che si dedica ad analizzare come le "norme" della lingua cambiano da cultura a cultura, non solo nel caso in cui il sistema linguistico utilizzato è diverso, ma anche quando, pur essendo lo stesso da un punto di vista formale, nella pratica non lo è, visto che è un'altra la cultura di riferimento.

Le differenze nel discorso e nelle strategie interazionali che, come già indicavano gli studi di Hymes (1962, 1972, 1974), definiscono l'identità culturale degli appartenenti a determinate lingue e culture, sono spesso fonte di incomprensione, anche perché un errore pragmatico (pragmatic failure, come lo ha definito Thomas, 1983) è di solito inteso come un "difetto" del carattere o della personalità - cosa che, tra l'altro, può causare o rafforzare stereotipi e pregiudizi - e non come mancanza di conoscenza o come risultato dello scontro tra aspettative, strategie e stili culturali diversi. Esistono, di fatto, schemi nel parlato delle comunità linguistiche che rimandano a fenomeni culturali e che sono in qualche modo identificabili e condivisi. Conoscerli può aiutare in questo senso e permetterci, non solo di comprendere meglio l'altro, ma anche di muoverci con maggiore consapevolezza (meta)pragmatica e di evitare conflitti.

Le ricerche nell'ambito della pragmatica cross-culturale hanno essenzialmente questo obiettivo. Un esempio ormai classico è il progetto Requests and Apologies: Cross-Cultural Speech Act Realization Patterns (CCSARP) (Blum-Kulka et al., 1989) che, come dice il titolo, si è occupato di due atti linguistici, le richieste e le scuse, confrontando lingue tra cui l'inglese, il francese, il tedesco e l'ebraico, per provare a scoprire in che modo si differenziavano tra loro e in quali aspetti presentavano eventuali somiglianze. Alcune lingue sono state tuttavia meno studiate di altre e l'italiano e il portoghese brasiliano, a cui ci dedicheremo in questo articolo, sono sicuramente tra queste.

Quelle con cui abbiamo iniziato sono alcune delle riflessioni da cui ha preso le mosse l'idea di un progetto, per molti versi comparabile a esperienze già fatte in precedenza (Blum-Kulka \& Olshtain 1984, Blum-Kulka et al. 1989, García 1989, Faerch \& Kasper 1989), che si propone di confrontare le caratteristiche di richieste appartenenti a diverse lingue e culture, partendo, in un primo momento, dall'identificazione dei mezzi linguistici utilizzati per la realizzazione di questo atto. Partecipano al progetto membri del gruppo di ricerca Pragmática (inter)linguística, intercultural e cross-cultural che è legato all'Universidade de São Paulo, in Brasile, e riunisce ricercatori che si occupano di varie lingue. Gli ambiti di ricerca del gruppo non si limitano alla pragmatica cross-culturale: si realizzano anche ricerche relative a una sola lingua e cultura (pragmatica intraculturale), poi eventualmente confrontata con le altre; a interazioni e confronti tra parlanti nativi e non nativi (pragmatica interculturale); o ancora all'insegnamento e all'apprendimento delle caratteristiche pragmatiche di una lingua e di una cultura da parte di non nativi (pragmatica interlinguistica) 1.

\footnotetext{
1 Una presentazione più dettagliata del gruppo di ricerca e dei suoi partecipanti si trova al link: http://pragmaticausp.weebly.com/.
} 


\section{normas}

Il gruppo si è inizialmente concentrato su portoghese brasiliano e italiano. Si sono più recentemente aggiunti lo spagnolo (per ora nelle varianti tuteante e voseante) e il tedesco, mentre è in preparazione il portoghese europeo. Sono state scelte metodologie di raccolta dei dati che potessero permettere di avere a disposizione un elevato numero di parlanti, in modo da identificare analogie, differenze e ricorrenze nelle diverse lingue.

Come si accennava, in questo articolo presentiamo un primo confronto tra dati dell'italiano e del portoghese brasiliano, a partire da un campione equilibrato per età, genere e provenienza di 30 parlanti nativi di italiano e 30 di portoghese brasiliano, con I'obiettivo di verificare, in modo esplorativo, come viene realizzata l'attenuazione nei due gruppi, utilizzando come strumento di analisi atti di supporto (supportive moves) e modificatori (modifiers), tanto a partire dall'insieme delle richieste analizzate, quanto tenendo conto di grado di imposizione (rank of imposition) e distanza sociale (social distance), seguendo due delle tre variabili della classica distinzione proposta da Brown e Levinson (1987), di cui parleremo nella sezione seguente.

\section{RICHIESTE E ATTENUAZIONE}

Le richieste, in quanto azioni che prevedono un "far fare" e che quindi determinano il comportamento futuro dell'interlocutore, sono atti linguistici in cui è frequente la comparsa dell'attenuazione (o mitigazione, come preferisce denominarla Caffi, 1999, 2001), offrendo quindi interessanti prospettive per un confronto tra diverse culture. Si tratta di atti direttivi che influiscono sia sul parlante che sull'interlocutore, visto che il richiedente si aspetta di vedere soddisfatta la sua richiesta, ma si espone, potendo anche ricevere una risposta negativa, mentre il destinatario della richiesta è obbligato a fornire una risposta e dovrà tendenzialmente giustificare la sua scelta, soprattutto nel caso in cui non voglia o non possa esaudire la richiesta. La richiesta è inoltre un classico Face Threatening Act (atto minaccioso per la faccia, FTA), secondo la definizione proposta da Brown e Levinson (1987), a partire dal concetto di faccia elaborato da Goffman (1967).

Sebbene si tratti di riferimenti molto noti, ricapitoliamo brevemente qui che, mentre la faccia positiva rimanda ai concetti di inclusione e appartenenza e si collega al bisogno di essere riconosciuti e di sentirsi parte di un gruppo, quella detta negativa si basa sull'idea di autonomia e individualità che si manifesta nel desiderio di libertà di azione, di non imposizione e di dominio del proprio territorio.

Il grado di minaccia della faccia viene modificato a partire da una serie di fattori e dobbiamo ancora a Brown e Levinson (1987: 76) la proposta di concentrarli su tre variabili chiamate distanza sociale, potere relativo e grado di imposizione. La distanza sociale tra gli interlocutori stabilisce un asse orizzontale; il potere relativo tra loro si organizza invece a partire da un asse verticale, quindi di tipo gerarchico, mentre il grado di imposizione è la relazione costo/beneficio che la realizzazione dell'atto linguistico rappresenta per gli interlocutori.

La possibilità di individuare nella richiesta, in maniera quasi prototipica, le variabili citate e $\mathrm{i}$ conseguenti fenomeni di attenuazione è probabilmente la ragione che ne ha fatto uno degli atti linguistici più frequentemente studiati. Analizzando una richiesta si possono, di fatto, studiare manifestazioni linguistiche che mostrano come un individuo o un gruppo 


\section{normas}

esprimono la loro sensibilità nei confronti di questi fattori e si può provare a capire che cosa modula il loro comportamento linguistico.

Tenendo conto di questa complessa rete di relazioni e del bisogno di "aggiustamento interazionale", la richiesta è un atto frequentemente associato alla cortesia linguistica intesa come I'insieme di «strutture ricorrenti nella lingua scritta e parlata che manifestano un comportamento comunicativo cooperativo e rispettoso» (Mariottini, 2007: 9) e quindi le norme e le convenzioni verbali adottate da una comunità per contenere la conflittualità nella relazione interpersonale, ma anche le strategie che determinano la scelta di certe forme linguistiche invece di altre al fine di consentire la negoziazione.

L'attenuazione fa parte di queste strategie così che spesso si associa alla cortesia o si fa addirittura coincidere con i procedimenti detti "cortesi", visto che le strategie utilizzate servono a ridurre i rischi collegati all'enunciazione e allontanano così conflitti, conseguenze sgradite o indesiderate e la possibilità di reazioni negative da parte dell'interlocutore.

Va detto tuttavia, riprendendo Caffi $(1999,2001)$ e Briz e Albelda (2013), che l'attenuazione non deve essere necessariamente legata alla cortesia, ovvero, alla tutela della relazione con l'altro. Intanto produce, in particolare nelle richieste, una diminuzione della forza illocutoria che ha in genere come obiettivo principale quello di far credere che si potrà ottenere più facilmente ciò che si chiede. Se voglio, per esempio, che qualcuno mi presti dei soldi perché ho dimenticato il portafogli, è preferibile che io lo faccia in modo "attenuato", utilizzando quindi elementi in grado di far apparire la mia richiesta meno incisiva e vincolante, grazie alla presenza di mezzi linguistici di diverso tipo. Sempre rimanendo nell'ambito delle richieste, possiamo anche pensare che, oltre a far aumentare le possibilità di vedere realizzata la propria richiesta, garantendo quindi lo scopo principale dell'interazione, l'attenuazione agisca anche sul versante della costruzione del sé, dando spazio a un bisogno identitario. In altre parole, potremmo dire che serve, da una parte, a soddisfare bisogni che potremmo definire "pratici", nel senso del raggiungimento di determinati obiettivi concreti, cosa particolarmente visibile nel caso delle richieste che prevedono l'immediato ottenimento di un riscontro, ma, dall'altra, si riferisce a quei bisogni che si possono chiamare relazionali, visto che attraverso la mitigazione si possono delineare contorni identitari, grazie ai quali il soggetto può costituirsi come un certo tipo di individuo, in modo molte volte corrispondente ai valori di una determinata lingua/cultura.

In particolare questo secondo aspetto rende rilevanti ricerche come quella che stiamo svolgendo, che ci consentono di osservare, a partire da dati empirici, che cosa fanno linguisticamente - o immaginano farebbero - dei parlanti nativi "reali" in determinate situazioni concretamente definite.

\section{LA METODOLOGIA DELLA RICERCA}

Saranno presentate di seguito le scelte metodologiche utilizzate per la raccolta e per l'analisi dei dati, particolamente importanti quando si fanno ricerche in prospettiva pragmatica, visto che delimitano, ancor più che in altri campi, il tipo di osservazioni che sarà possibile fare e i risultati che si potranno ottenere. 


\subsection{La raccolta dei dati}

Per la raccolta dei dati è stato utilizzato un questionario fornito elettronicamente ai partecipanti tramite la piattaforma Google $D_{r i v e}^{2}$ e diviso in due parti: (1) una prima parte composta da DCT (Discourse Completion Test) scritti e aperti (viene descritta una situazione e si chiede all'informatore di scrivere ciò che direbbe a partire dalle indicazioni date); (2) una seconda parte costruita con il sistema della scelta multipla (dopo la situazione vengono proposte opzioni tra cui scegliere). Prima di iniziare a compilare le due parti del questionario, al partecipante viene richiesto che fornisca dati "biolinguistici" come età, professione, formazione, provenienza, lingue conosciute, ecc. $\mathrm{Ci}$ dedicheremo qui esclusivamente alla parte del questionario composta da DCT. ${ }^{3}$

Nella formulazione dei DCT si è tenuto conto di alcuni fattori, tra cui, ad esempio, che le situazioni comunicative proposte avvengono in due tipi di ambientazione, suddivise equamente tra ambito pubblico (per strada) e ambito privato (nella propria casa o in quella di altri). Tuttavia, le variabili che prenderemo in considerazione in questa sede sono solo due: grado di imposizione e distanza sociale.

I DCT - scritti e detti per questo anche WDCT (Written Discourse Completion Test) - sono stati ideati dal gruppo di ricerca che ha dedicato riflessione attenta alla loro elaborazione. La decisione di lasciarli aperti, ovvero di limitarsi a descrivere una situazione senza prevedere uno script nel quale mancasse un solo turno, è dipesa dal fatto che si è voluta lasciare ai partecipanti la possibilità di scegliere che cosa dire, così come sarebbe avvenuto nella vita "reale", ovvero senza conoscere I'esito della richiesta realizzata. Vediamo di seguito le otto situazioni:

\footnotetext{
2 II questionario in italiano si trova al link: http://migre.me/vVo3K, mentre quello in portoghese brasiliano: http://migre.me/vVo10.

${ }^{3}$ Sebbene non sia oggetto di questo articolo, descriviamo qui brevemente anche la parte del questionario a scelta multipla che offriva ai partecipanti cinque opzioni basate su una suddivisione proposta da Haverkate (1994), a sua volta, ripresa dallo schema di Brown \& Levinson (1987): (a) si può evitare la richiesta (ci si allude senza che venga citata - off record); (b) si può realizzare la richiesta in modo indiretto; (c) si può realizzare la richiesta mostrando la cosiddetta cortesia negativa; (d) si può realizzare la richiesta mostrando la cosiddetta cortesia positiva; (e) si può realizzare la richiesta in modo diretto e senza manifestazioni di cortesia.
} 
Tabella 1 - Le situazioni proposte ai parlanti nativi di italiano (IT) e portoghese brasiliano $(\mathrm{PB})$

\begin{tabular}{|c|c|c|}
\hline Situazione & $\begin{array}{l}\text { Grado di } \\
\text { imposizione } \\
\text { (alto } \uparrow / \text { basso } \downarrow \text { ) }\end{array}$ & $\begin{array}{l}\text { Distanza } \\
\text { sociale (alta } \\
\uparrow / \text { bassa } \\
\downarrow \text { ) }\end{array}$ \\
\hline $\begin{array}{l}\text { 1. Tu e un tuo amico/una tua amica siete appena saliti(-e) } \\
\text { sull'autobus quando ti accorgi che hai dimenticato il portafoglio } \\
\text { e non puoi fare il biglietto. Che cosa dici? } \\
\text { 1. Você e um(a) amigo(a) entram em um ônibus e, nesse } \\
\text { momento, você percebe que esqueceu a carteira e não tem } \\
\text { como pagar a passagem. O que você Ihe diz? }\end{array}$ & $\downarrow$ & $\downarrow$ \\
\hline $\begin{array}{l}\text { 2. Devi andare in aeroporto e sei in ritardo. Al posteggio dei taxi, } \\
\text { una persona che sembra avere la tua età è in fila e aspetta per } \\
\text { prendere il prossimo taxi... Che cosa dici? } \\
\text { 2. Você tem urgência para chegar ao aeroporto. Na fila do ponto } \\
\text { de táxi, há uma pessoa da sua idade na frente. O que você Ihe } \\
\text { diz? }\end{array}$ & $\uparrow$ & $\downarrow$ \\
\hline $\begin{array}{l}\text { 3. Sei per strada e vuoi scrivere il numero di telefono di un } \\
\text { annuncio che vedi passando. Ti accorgi, però, di non avere una } \\
\text { penna con te. Passa un signore... Che cosa gli dici? } \\
\text { 3. Você está andando na rua e precisa anotar o número de } \\
\text { telefone de um anúncio que viu. Percebe, porém, que não tem } \\
\text { caneta. Um senhor está passando... O que você Ihe diz? }\end{array}$ & $\downarrow$ & $\uparrow$ \\
\hline $\begin{array}{l}\text { 4. Sei per strada, devi incontrare un tuo amico straniero che sta } \\
\text { per arrivare dopo un lungo viaggio e sarà ospite a casa tua. A } \\
\text { causa di un imprevisto, arriverai in ritardo e devi avvisarlo. Il tuo } \\
\text { cellulare per qualche inspiegabile motivo non funziona e non ci } \\
\text { sono neanche telefoni pubblici in giro. Non ti resta che cercare } \\
\text { un cellulare in prestito per una telefonata e c'è un signore che } \\
\text { sta passando... Che cosa gli dici? } \\
\text { 4. Você está na rua indo encontrar um amigo estrangeiro, que } \\
\text { está chegando de viagem. Por causa de um imprevisto, vai se } \\
\text { atrasar e precisa avisá-lo. Seu celular, por algum motivo, não } \\
\text { funciona. Também não há telefones públicos ao redor. Assim, } \\
\text { sua única opção é que o senhor que está passando Ihe } \\
\text { empreste um celular... O que você Ihe diz? }\end{array}$ & $\uparrow$ & $\uparrow$ \\
\hline $\begin{array}{l}\text { 5. Sei appena entrato a casa di un tuo amico/una tua amica e } \\
\text { hai sete. Che cosa dici? } \\
\text { 5. Você acabou de entrar na casa de um(a) amigo(a) e está com } \\
\text { sede. O que você Ihe diz? }\end{array}$ & $\downarrow$ & $\downarrow$ \\
\hline $\begin{array}{l}\text { 6. Sei appena entrato a casa di un tuo amico/una tua amica } \\
\text { bagnato fradicio perché fuori sta piovendo a dirotto. Hai bisogno } \\
\text { di farti una doccia o almeno di toglierti i vestiti bagnati... Che } \\
\text { cosa dici? } \\
\text { 6. Você acabou de entrar na casa de um(a) amigo(a) e está } \\
\text { completamente encharcado(a) porque do lado de fora chove } \\
\text { torrencialmente, por isso, precisa tomar um banho ou ao menos } \\
\text { trocar as roupas molhadas... O que você Ihe diz? }\end{array}$ & $\uparrow$ & $\downarrow$ \\
\hline $\begin{array}{l}\text { 7. Sei andato con un tuo amico/una tua amica a cena a casa di } \\
\text { suoi colleghi di lavoro che fino a quel momento non conoscevi. }\end{array}$ & $\downarrow$ & $\uparrow$ \\
\hline
\end{tabular}


Prima di cominciare a mangiare, ti rendi conto che dove ti sei seduto manca la forchetta. Che cosa dici ai padroni di casa? 7. Você acompanha um amigo a um jantar na casa de alguns colegas, que até aquela ocasião você não conhecia. Ao iniciar a refeição, percebe que no lugar onde sentou falta o garfo. 0 que você diz aos donos da casa?

8. Ti sei appena trasferito in un nuovo appartamento. Durante le prime due settimane, noti che la signora del piano di sopra fa un rumore eccessivo, anche al di fuori dell'orario in cui è consentito. Decidi di parlare con lei per cercare di risolvere il problema. Che cosa le dici?

8. Você mudou recentemente para um novo apartamento. Durante as duas primeiras semanas, percebe que a senhora do andar de cima faz barulho excessivo, mesmo fora do horário permitido. Decide conversar com ela para resolver o problema. O que você Ihe diz? 


\section{normas}

Nella descrizione della situazione si indicano elementi che permettano di definire un contesto, tra cui il luogo, l'età dell' interlocutore (presupponendo che chi risponde la metterà in relazione con la sua età reale), il tipo di rapporto (dall'amico al passante, dal coetaneo alla persona più anziana) e la motivazione o il bisogno da cui ha origjne la richiesta. Si è avuta cura di non usare il verbo "richiedere"/"pedir" per elicitare la richiesta, ma di usare un più generico "Che cosa dici?"/"O que você Ihe diz?" per non influenzare la risposta e, al tempo stesso, per condurre all'utilizzo del discorso diretto. L'altro fattore di cui si è tenuto conto al momento della formulazione della richiesta è stato che si proponessero situazioni in cui chiunque potrebbe trovarsi senza bisogno di immaginare un'identità, un'età o una posizione sociale diversa dalla propria. Non si chiede in nessun caso di assumere un ruolo, ma semplicemente di immaginare se stessi in una data situazione comunicativa ed è per questo che la variabile potere relativo è stata esclusa.

Un breve chiarimento sulla scelta della metodologia per la raccolta dei dati per la quale è fondamentale pensare agli obiettivi e alle condizioni di ogni ricerca. Come si è visto, le metodologie che abbiamo scelto sono scritte e tra quelle che si definiscono "controllate", ovvero quelle con elevata validità interna (Pallotti, 2001; Mackey \& Gass, 2005; FélixBrasdefer, 2010; Santoro, 2012), che offrono dei vantaggi al ricercatore, pur avendo alcuni svantaggi. Sappiamo, per esempio, che lo scritto fa perdere i tratti dell'oralità e la spontaneità, visto che chi partecipa alla ricerca scrive e ha normalmente il tempo di pensare e magari anche di cancellare, tornare indietro e riscrivere. È ovvio, inoltre, che questo tipo di metodologia non permette l'interazione. D'altro canto, però, raccogliere i dati in questo modo consente di avere a disposizione un numero anche molto elevato di informatori, cosa decisamente più difficile nel caso di registrazioni audio e/o video. In tal modo, aumenta il grado di interpretabilità perché si hanno più elementi per dire se le variazioni che si riscontrano sono effettivamente determinate dalle variabili selezionate; è possibile identificare ricorrenze che fanno emergere fenomeni o ci possono aiutare a confermare o smentire ipotesi; si possono ottenere informazioni sul sapere pragmatico utilizzato nello svolgimento di determinate pratiche comunicative. Inoltre, questo tipo di ricerca, essendo ampliabile e replicabile, permette non solo di raccogliere dati di più lingue, ma anche di ripetere l'esperimento a distanza di tempo.

\subsection{La metodologia di analisi}

Oltre al fatto che le domande a scelta multipla non sono l'oggetto scelto per questo articolo, è piuttosto semplice osservarne i risultati, visto che la piattaforma utilizzata per divulgare il questionario online permette di creare automaticamente grafici e fornisce un resoconto di tutte le risposte ricevute.

Più complessa è invece l'analisi delle risposte aperte. Come si è detto, ognuno dei partecipanti invia otto risposte, scrivendo ciò che direbbe in ciascuna delle situazioni proposte. Ci si trova quindi di fronte a enunciati sempre diversi tra loro ed è quindi indispensabile che vengano osservati uno per uno, selezionando categorie di analisi.

Il primo passo per poter procedere a una prima analisi contrastiva dei risultati nelle due lingue è stato selezionare tra le risposte pervenute quelle di parlanti che potessero costituire un campione equilibrato e comparabile. II campione sulle cui risposte ci baseremo qui ha le seguenti caratteristiche: 
Tabella 2 - II campione utilizzato per la ricerca

\begin{tabular}{|c|c|c|c|}
\hline & $\begin{array}{ll}\begin{array}{l}\text { Parlanti } \\
\text { di italiano }\end{array} & \text { nativi } \\
\end{array}$ & $\begin{array}{l}\text { Parlanti nativi } \\
\text { di portoghese brasiliano }\end{array}$ \\
\hline \multicolumn{2}{|c|}{ Numero di partecipanti } & 30 & 30 \\
\hline \multirow[t]{4}{*}{ ETÀ } & $18-30$ & 13 & 14 \\
\hline & $31-40$ & 3 & 5 \\
\hline & $41-50$ & 7 & 5 \\
\hline & $>51$ & 7 & 6 \\
\hline \multirow[t]{2}{*}{ Genere } & $F$ & 22 & 19 \\
\hline & $M$ & 8 & 11 \\
\hline \multicolumn{2}{|c|}{ Provenienza } & $\begin{array}{l}10 \text { Nord, } 10 \text { Centro, } 10 \text { Sud e } \\
\text { isole }\end{array}$ & San Paolo e dintorni \\
\hline
\end{tabular}

Le categorie utilizzate per l'analisi dell'attenuazione nelle richieste in questo studio sono le seguenti: (a) sottoatti o atti di supporto (supportive moves), separati dal nucleo o atto principale (head act) e classificati in base alla loro funzione; (b) modificatori morfosintattici, lessicali e discorsivi, selezionati e raggruppati in base alla strategia linguistica utilizzata.

Nonostante sia sempre molto difficile separare ed evitare accavallamenti, ci siamo sforzati di elaborare un primo modello, ispirandoci alla classificazione proposta da Blum-Kulka et al. (1989) e già adattata per l'italiano da Nuzzo (2007) e partendo dal corpus concreto che ci apprestavamo ad analizzare. Va detto che sono stati discussi in maniera dettagliata e approfondita i dubbi e le diverse interpretazioni, ma che si è deciso alla fine di mettere alla prova questa categorizzazione che, rispetto ad altre possibili, ha il vantaggio di essere stata pensata specificamente per le richieste e di non avere un numero eccessivo di categorie.

In particolare, può sembrare contraddittorio che ci sia una categoria che abbiamo chiamato "marca di cortesia", pur avendo chiarito all'inizio che non crediamo che l'attenuazione si debba confondere con la cortesia perché siamo convinti che i suoi scopi vanno oltre il semplice effetto della perdita o della protezione della faccia, come si è spesso creduto. La decisione di utilizzare comunque questa denominazione deriva dal fatto che si tratta del modo classico in cui vengono denominate formule ritualizzate e standardizzate come ad esempio "per favore" o "gentilmente" per l'italiano e por favor o por gentileza per il portoghese brasiliano. A questo proposito, è importante sottolineare che l'annotazione del corpus, che descriveremo meglio più avanti, essendo fatta manualmente, permette di identificare la funzione di ciascuna delle strategie. Nel caso di un elemento linguistico come por favor del portoghese brasiliano, solo per fare un esempio, l'annotazione avverrà in base alla sua effettiva funzione che può essere di marca di cortesia, ma anche di appello, visto che può essere anche utilizzato per richiamare l'attenzione dell'interlocutore.

Un'altra categoria che potrebbe apparire inadeguata al contesto di analisi che ci proponiamo è quella dei rafforzatori che hanno l'obiettivo di mostrare I'urgenza e/o l'assoluta necessità della richiesta effettuata e che abbiamo lasciato tra i modificatori lessicali. In questo caso, la decisione di considerare anche questa come una possibile forma di "attenuazione" è dipesa dal fatto che la sua presenza ha in qualche modo la funzione di giustificare riducendo i rischi e l'assunzione di responsabilità da parte di chi richiede che, dicendo di trovarsi in una situazione di emergenza, spiega perché si rivolge all'altro. 


\section{normas}

La categorizzazione che vedremo è stata messa alla prova con questo campione anche per definire quali atti di supporto (<as $>$ ) e modificatori sono meno produttivi, in modo che si possa pensare ad eventuali adattamenti per future ricerche ed approfondimenti.

Gli atti di supporto che sono stati presi in considerazione sono i seguenti (la sigla tra parentesi è quella utilizzata per l'annotazione del corpus):

- Appello (<as1>) che serve a stabilire un primo contatto con l'interlocutore prima che sia formulata la richiesta e per il quale vengono usati nomi propri, nomi affettivi o altri mezzi linguistici che richiamino l'attenzione dell'interlocutore (IT - mi scusi/scusami, senta/senti, buongiorno; signore/signora/signorina; ciao; salve; PB - por favor, com licença; moço, senhor, amigo, bom dia, olá; desculpe).

- Esclamazione (<as2>) che si usa per esprimere sorpresa, contrarietà, delusione, ecc. e può creare empatia iniziale con l'interlocutore (IT - caspita!, mannaggia!, porca miseria!; PB = nossa!, vixi!, putz!).

- Minimizzatore (<as3>) che produce come effetto l'idea della diminuzione dello sforzo o del costo della richiesta (IT - non ci vogliono più di 5 minuti; solo se anche lei va in quella direzione...; PB - não vai demorar mais que 5 minutos...; só se você também for para lá...).

- Preparatore (<as4>) che annuncia immediatamente la richiesta con l'obiettivo di ridurre le possibilità di rifiuto, eventualmente mostrando che il parlante è consapevole del costo della richiesta; si noti che per ottenere questo fine preparatorio si può anche mettere in evidenza un difetto o una mancanza del parlante, presentandolo come responsabile della situazione che lo porta a realizzare la richiesta (IT - ti devo chiedere un grosso favore...; come sempre, mi sono dimenticato...; PB - preciso pedir um grande favor...; como sempre acontece comigo, me esqueci...).

- Giustificazione (<as5>) che evidenzia la motivazione o il fine per il quale viene realizzata la richiesta (IT - Ho dimenticato il portafoglio a casa...; ... che mi devo asciugare; Esqueci minha carteira em casa...; PB - ...para me enxugar).

- Proposta di risarcimento o garanzia (<as6>) con cui si offre all'interlocutore qualcosa in cambio di ciò che fa/farà o si garantisce che la perdita momentanea sarà risarcita (IT posso usare il tuo telefono? ti pago la telefonata...; mi presti il tuo libro? giuro che te lo ridò domani...; PB - me permite usar seu telefone? eu pago a ligação...; você me empresta seu livro? juro que amanhã devolvo...).

- Proposta di alternative (<as7>) con cui si offre un'alternativa all'interlocutore, escludendo o minimizzando le conseguenze negative della non realizzazione dell'atto, a volte con I'uso di strutture condizionali e temporali (IT - se non puoi, non fa niente...; quando puoi, mi presti il tuo computer?; PB - se você não puder, eu dou um jeito...; quando puder, você me empresta seu computador?).

- Adulatore $(<a s 8>)$ che mette in risalto aspetti positivi dell'interlocutore (gentilezza, disponibilità, ecc.) con l'obiettivo di persuaderlo a fare quanto viene richiesto (IT - sarebbe così gentile da...; tu che mi risolvi sempre i problemi...; PB = você faria a gentileza de...; você que sempre me ajuda...). 


\section{normas}

Richieste e attenuazione | Elisabetta Santoro

- Ringraziamento (<as9>) che conclude la sequenza dell'interazione iniziata con la richiesta (IT - grazie; ti/La ringrazio; PB - obrigado/-a; agradeço).

- Riconoscimento del costo della richiesta (<as10>) che mostra che il parlante è consapevole del costo della richiesta (IT - se non ti scoccia; ti secca se...; se non è chiedere troppo; so che per te non è facile, ma ti devo proprio chiedere...; PB - se não for incômodo...; se não for abuso...; se não for pedir demais; eu sei que para você não é fácil, mas precisaria realmente...).

In ognuna delle risposte abbiamo identificato gli atti di supporto presenti, limitandoci in questo primo esperimento alla classificazione e successiva verifica del numero e del tipo di strategie linguistiche considerate attenuative. È evidente che anche la presenza o assenza dell'atto principale e la classificazione del modo più o meno indiretto in cui viene espresso sono elementi da considerare parte dei procedimenti di attenuazione, a cui, tuttavia, ci dedicheremo in altra sede.

Oltre agli atti di supporto, abbiamo selezionato un numero ridotto di modificatori a partire dalle strategie presenti nel nostro corpus che hanno essenzialmente come obiettivo creare l'illusione di un distanziamento rispetto alla richiesta o cercano di farla apparire meno costosa o vincolante. Per i modificatori è stata proposta una tripartizione in morfosintattici, lessicali e discorsivi e, come si vedrà a seguire, per ognuna di queste tre categorie ci sono ulteriori suddivisioni.

II primo gruppo è quello dei modificatori morfosintattici $(<m m s>$ ) (dopo ciascun elemento, abbiamo riportato la sigla utilizzata per l'annotazione):

(a) Dislocamento verbale $<m m s 1>$ con cui il parlante realizza l'atto producendo uno spostamento dell'asse deittico temporale o del modo; in italiano e portoghese brasiliano, si tratta perlopiù di usi di condizionale e imperfetto (IT - ci sarebbe...; volevo chiedere...; PB haveria...; queria perguntar...?).

(b) Verbi modali e costruzioni equivalenti $<m m s 2>$ che si riferiscono alla modalità deontica, esprimendo il senso di obbligo, proibizione, necessità, possibilità, permesso, ecc., (IT - dovrei andare con una certa urgenza in aeroporto; mi posso fare una doccia?; è possibile avere una forchetta?; PB - deveria ir com urgência até o aeroporto; posso tomar um banho? dá para pegar um garfo?) o la volontà.

(c) Incassatura $<\mathrm{mms} 3>$ che compare come elemento introduttivo della richiesta con il frequente uso di condizionale o imperfetto (IT - volevo sapere se...; le sarei grata/-o se; PB - queria saber se...; agradeceria se...).

(d) Negazione dell'interrogativa o formule dubitative $<m m s 4>$ che, inserita nella domanda, sembra avere la funzione di verifica o conferma, come se il richiedente dubitasse del fatto che la richiesta possa essere soddisfatta (IT - non mi puoi mica prestare...?; non è che...; non so se...; PB - você não pode me emprestar...? não sei se...; será que...)

(e) Diminutivi $<m m s 5>$ che creano l'illusione che ciò che viene richiesto non comporterà un grande sforzo per l'interlocutore (IT - solo un attimino...; PB - só um minutinho...; me faz um favorzinho). 
I modificatori lessicali sono:

(a) Minimizzatori o indeterminatori lessicali $<\mathrm{ml} 1>$ che trasmettono l'idea della riduzione o dell'indeterminazione dello sforzo necessario (IT - un po', un attimo...; in linea di massima; diciamo...; hai un po' di soldi da darmi?; mi sa che...; PB - um pouco, um momento, em princípio...; você tem algum dinheiro para me dar?; acho que...).

(b) Dubitatori lessicali $<\mathrm{ml} 2>$ che indicano un dubbio circa la realizzazione dell'atto, diminuendo così le aspettative (IT - per caso; forse; PB - por acaso; talvez).

(c) Rafforzatori lessicali <ml3> che enfatizzano l'importanza e l'urgenza dell'atto, giustificandolo e presentandolo quasi come inevitabile (IT - urgentemente; davvero; proprio; PB - com urgência, realmente).

(d) Scelte lessicali attenuanti $<\mathrm{ml} 4>$ che, essendo meno dirette, possono rappresentare un maggior grado di distanziamento rispetto all'interlocutore e rispetto alla richiesta (IT - mi permette/mi consente..?; le dispiace se... ?; mi procura un portacenere...?; PB - me permite...?; você se incomoda se... ?; você me arruma...?; você tem como...?).

Ci sono poi quelli che abbiamo definito modificatori discorsivi perché si inseriscono nella sequenza del discorso:

(a) Attivatori di vincolo o empatia <md1> che stabiliscono un contatto diretto con l'interlocutore per avvicinarlo al parlante; appartengono a questa categoria marcatori fatici, richieste di accordo e altre espressioni che creano empatia (IT - sai, vedi; non è vero? non ti pare? me lo faresti questo favore?; PB - sabe, veja; não é verdade? você não acha? você faria isso pra mim?).

(b) Marche di cortesia $<m d 2>$ che vengono usate quando si ricorre direttamente alla collaborazione dell'interlocutore (IT - per favore; dai; gentilmente; per cortesia; cortesemente; PB - por favor; vai; gentilmente; por gentileza).

(c) Riformulatori $<m d 3>$ che manifestano esitazione e mostrano che la formulazione dell'atto crea un certo disagio al parlante (in posizione finale, possono precedere una sospensione) (IT - cioè; insomma; allora; PB - quer dizer; enfim; então).

In questa prima analisi, non abbiamo distinto tra modificatori interni (quelli inseriti nell'atto principale) e modificatori esterni (che invece si trovano al di fuori dell'atto principale), visto che l'obiettivo era essenzialmente verificare se un determinato tipo di modificatore veniva preferito rispetto ad altri e se era possibile constatare analogie e differenze comparando le due lingue da questo punto di vista.

Una volta definita la tipologia di atti di supporto e modificatori con valore attenuativo che sarebbero stati usati in questa fase della ricerca, siamo passati all'annotazione del corpus, realizzata manualmente, dopo aver copiato e selezionato le risposte in un file .txt, come quello riprodotto di seguito, a partire dal quale è stata identificata e annotata con le sigle prescelte la presenza di elementi attenuatori con un segnale di apertura (<sigla $>$ ) all'inizio di ogni espressione da annotare e uno di chiusura $(</$ sigla $>$ ) alla fine, affinché si potesse 
sempre vedere dove esattamente inizia e dove finisce ciascun elemento ${ }^{1}$. Vediamo nella riproduzione un esempio di annotazione:

Figura 1 - Un esempio di richieste annotate in un file .txt

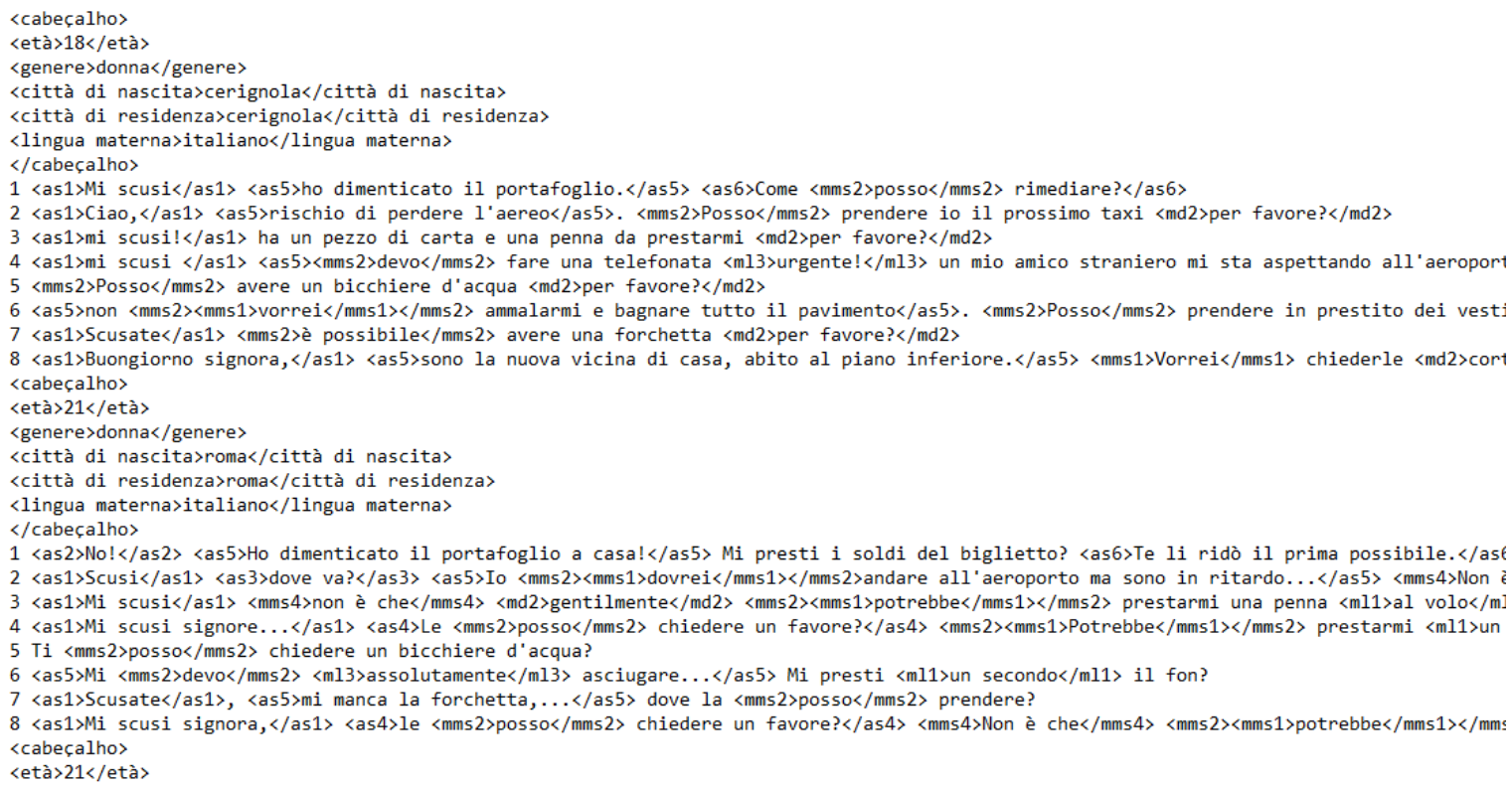

Nell'intestazione (cabeçalho) che precede le otto risposte di ciascun informante sono stati riportati i suoi principali dati "biolinguistici", in modo da permettere di verificare in qualsiasi momento a chi appartiene la risposta che si sta esaminando. In ognuna delle risposte sono poi stati identificati e annotati gli elementi attenuativi. Una volta conclusa I'annotazione, è possibile con facilità interrogare il corpus con il software AntConc, scaricabile gratuitamente online e utilizzato nella linguistica dei corpora, con il quale sono stati fatti i primi conteggi ed esperimenti.

Vediamo, a seguire, a titolo di esempio, la schermata di AntConc nel momento in cui abbiamo inserito per la ricerca la sigla dell'atto di supporto appello (<as $1></ a s 1>)$ per sapere il numero delle occorrenze in italiano.

\footnotetext{
${ }^{1}$ Ringrazio Mayara da Silva Neto, studentessa della Scuola di master e dottorato dell'Universidade de São Paulo e membro del gruppo di ricerca, per la collaborazione per l'annotazione del corpus.
} 


\section{normas}

Figura 2 - schermata di AntConc - ricerca appello </as1 $>$ in italiano (IT)

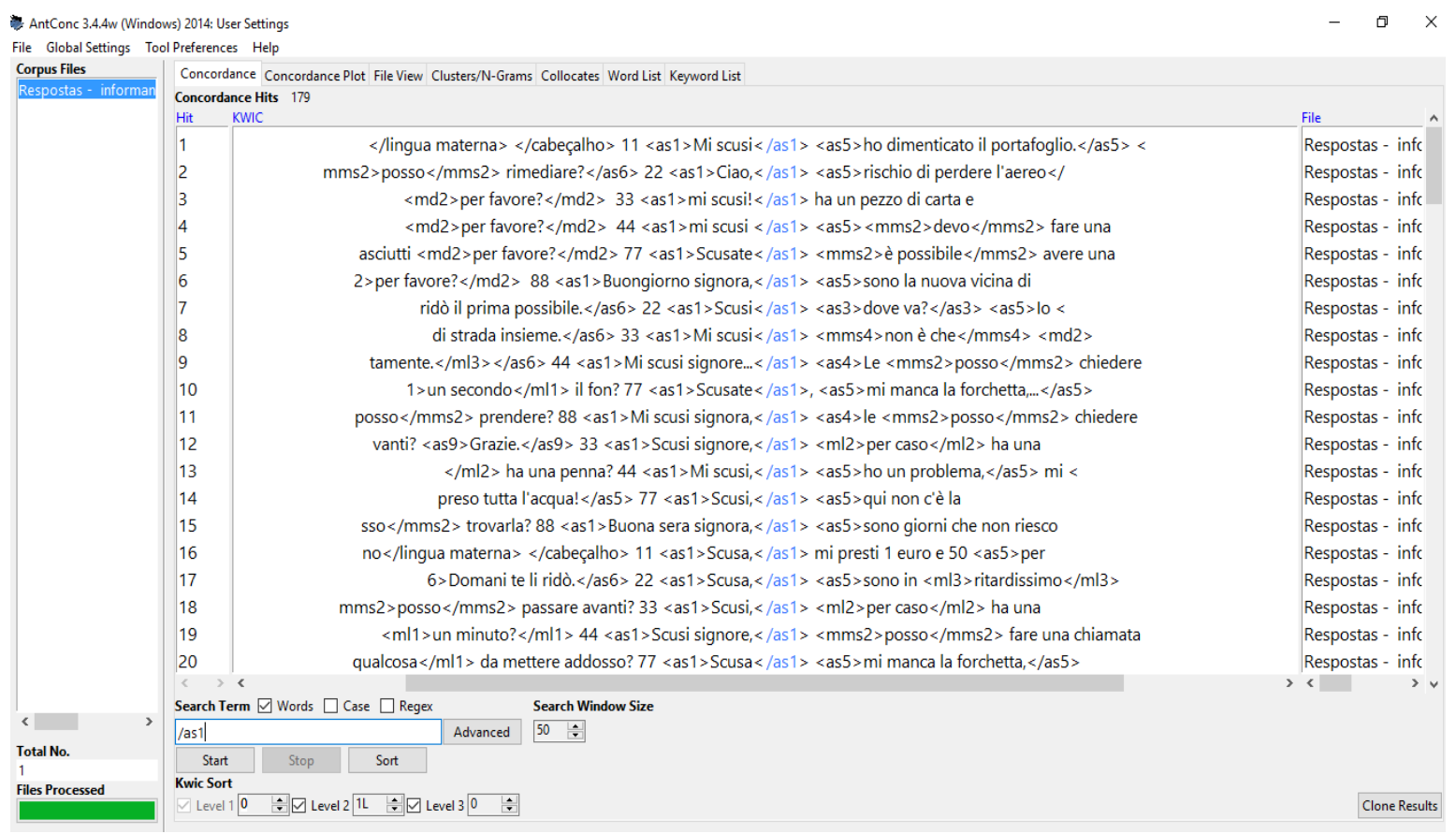

In azzurro compare la sigla che è stata inserita per dare inizio alla ricerca. Nell'esempio si tratta della sigla </as1> che indica la chiusura dell'etichetta utilizzata per l'appello. In alto a sinistra si trova il numero di occorrenze (concordance hits) che in questo caso sono 179. A sinistra della sigla si vede invece l'elemento che è stato annotato, cosa che permette di visualizzare immediatamente quali sono i mezzi linguistici utilizzati per la realizzazione della strategia (si noti comunque che ogni schermata contiene solo 20 risultati e che per vedere il resto sarà necessario scorrere in avanti). In questo caso, si osserva che in italiano l'appello è stato prevalentemente espresso con l'imperativo del verbo scusare alla seconda persona singolare (scusa) e plurale (scusate) e alla terza persona singolare, dove viene spesso preceduto dal pronome personale mi (scusi/mi scusi). II numero che compare raddoppiato nella stringa corrisponde al numero della situazione a cui si riferisce la richiesta, cosa che, conoscendo le otto situazioni, permette di sapere qual era il contesto in cui sono stati utilizzati l'atto di supporto o il modificatore che si sta osservando. In tal modo, è possibile anche sapere qual è la posizione dell'elemento cercato, così che, se per esempio si trova subito dopo il numero come qui, sappiamo che l'elemento annotato si trova all'inizio della richiesta. Può essere utile a capire la struttura delle richieste analizzate anche osservare che cosa segue l'elemento che è stato cercato. Nella schermata che stiamo usando come esempio in 10 casi su 20 segue la sigla </as5> che indica la presenza di una giustificazione. 
Sotto, sempre a titolo di esempio, la schermata di AntConc relativa al portoghese brasiliano in risposta alla stessa interrogazione, eseguita tramite l'inserimento della sigla $</$ as $1>$ che, come si è visto, indica la chiusura dell'etichetta relativa all'appello che compare in azzurro.

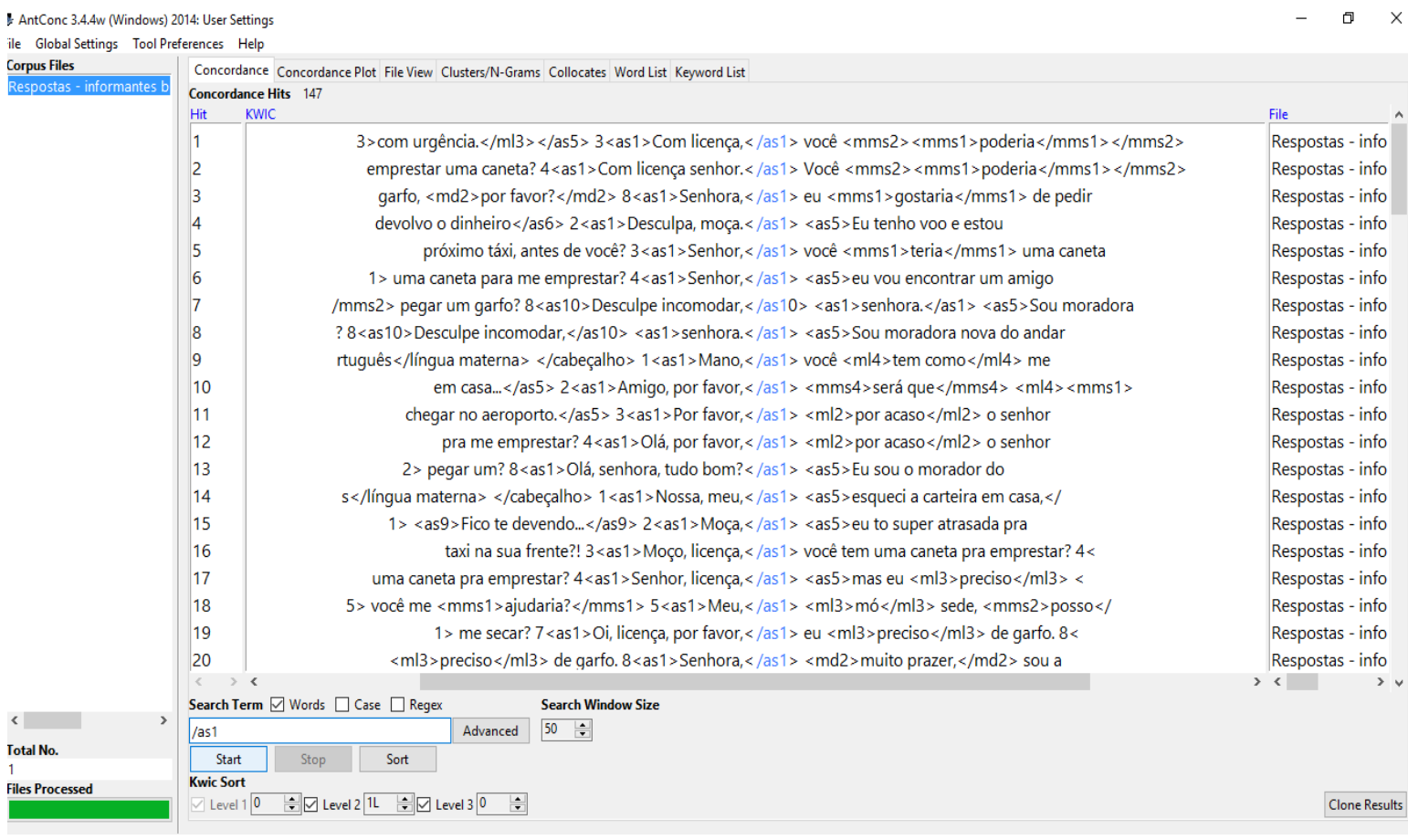

Figura 3 - schermata di AntConc - ricerca appello </as1 > in portoghese brasiliano (PB)

Anche qui si vede in alto a destra il numero di occorrenze (in questo caso 147). In azzurro, come nella schermata precedente, compare l'etichetta utilizzata per la ricerca, alla cui sinistra si possono visualizzare gli elementi che hanno motivato l'annotazione. Nella schermata che abbiamo riprodotto qui i più frequenti sono (com) licença, diverse volte accompagnato da un vocativo espresso tramite senhor/senhora/moço o altri appellativi che si riferiscono all'interlocutore, oltre a por favor, usato qui evidentemente non come marca di cortesia, ma come mezzo per richiamare l'attenzione. In queste richieste, 7 volte su 20 all'appello iniziale segue una giustificazione.

\section{I RISULTATI: ANALISI E DISCUSSIONE}

Passiamo ora ai risultati di questa prima fase del lavoro di ricerca che, come si è detto, ha carattere esplorativo, visto che il principale obiettivo è mettere alla prova le categorie selezionate, in ciascuna delle due lingue e nel confronto fra entrambe, e le variabili a partire dalle quali si è deciso di valutare se possono essere ritenute sensibili.

L'osservazione delle strategie riconoscibili e la verifica di analogie e differenze della loro presenza nelle due lingue partono da risultati quantitativi sulla base dei quali verranno fatte le prime riflessioni. Inizieremo dai risultati riportati nella tabella 3 che contiene il numero di occorrenze di ciascuna tipologia di atto di supporto nel campione di parlanti nativi di italiano e in quello di parlanti nativi di portoghese brasiliano, seguita dalla percentuale calcolata sulla base del numero totale di 240 risposte per ognuno dei due campioni. 
Tabella 3 - Atti di supporto nel corpus in italiano (IT) e portoghese brasiliano (PB)

\begin{tabular}{|c|c|c|c|}
\hline \multirow[b]{2}{*}{ Tipologia di atto di supporto } & \multirow[b]{2}{*}{ Sigla } & \multicolumn{2}{|c|}{ Numero occorrenze } \\
\hline & & IT (240) & PB (240) \\
\hline Appello & $<\mathrm{as} 1>$ & $\begin{array}{l}179 \\
(74,58 \%)\end{array}$ & $\begin{array}{l}147 \\
(61,25 \%)\end{array}$ \\
\hline Esclamazione & $<\operatorname{as} 2>$ & $\begin{array}{l}15 \\
(6,25 \%)\end{array}$ & $\begin{array}{l}20 \\
(8,33 \%)\end{array}$ \\
\hline Minimizzatore & $<$ as3 $>$ & $\begin{array}{l}21 \\
(8,75 \%)\end{array}$ & $\begin{array}{l}13 \\
(5,41 \%)\end{array}$ \\
\hline Preparatore & $<\mathrm{as} 4>$ & $\begin{array}{l}17 \\
(7,08 \%)\end{array}$ & $\begin{array}{l}13 \\
(5,41 \%)\end{array}$ \\
\hline Giustificazione & $<$ as5> & $\begin{array}{l}171 \\
(71,25 \%)\end{array}$ & $\begin{array}{l}152 \\
(63,33 \%)\end{array}$ \\
\hline Proposta di risarcimento o garanzia & $<$ as6> & $\begin{array}{l}19 \\
(7,91 \%)\end{array}$ & $\begin{array}{l}25 \\
(10,41 \%)\end{array}$ \\
\hline Proposta di alternative & $<\operatorname{as} 7>$ & $\begin{array}{l}1 \\
(0,41 \%)\end{array}$ & $\begin{array}{l}1 \\
(0,41 \%)\end{array}$ \\
\hline Adulatore & $<\operatorname{as} 8>$ & $\begin{array}{l}16 \\
(6,66 \%)\end{array}$ & $\begin{array}{l}3 \\
(1,25 \%)\end{array}$ \\
\hline Ringraziamento & $<$ as9> & $\begin{array}{l}13 \\
(5,41 \%)\end{array}$ & $\begin{array}{l}13 \\
(5,41 \%)\end{array}$ \\
\hline Riconoscimento del costo della richiesta & $<$ as10 $>$ & $\begin{array}{l}36 \\
(15 \%)\end{array}$ & $\begin{array}{l}23 \\
(9,58 \%)\end{array}$ \\
\hline
\end{tabular}

II risultato evidenzia una tendenza simile nelle due lingue, sebbene sia presente una differenza percentuale significativa: sia in italiano che in portoghese brasiliano i due atti di supporto con una frequenza di gran lunga predominante rispetto a tutti gli altri sono I'appello, presente in 179 su 240 casi (74,58\%) nelle richieste in italiano (IT) e in 147 su 240 $(61,25 \%)$ in portoghese brasiliano (PB), e la giustificazione con 171 occorrenze in italiano $(71,25 \%)$ e 152 in portoghese (63,33\%). In altre parole, nel campione analizzato, I'assoluta maggioranza dei parlanti nativi sceglie di iniziare stabilendo un contatto con l'interlocutore. Se si considera che le richieste sono state scritte e non sono registrazioni spontanee, il dato ha sicuramente una particolare rilevanza e indica che nella competenza metapragmatica degli informatori è considerato essenziale partire da questa "mossa" prima di passare alla richiesta vera e propria. Questa capacità di riprodurre una situazione interazionale viene rafforzata dalla presenza di esclamazioni che, sebbene con un numero di occorrenze non molto elevato, sono comunque presenti nelle richieste analizzate. Ancora un aspetto conferma l'idea che i parlanti hanno in qualche modo incluso la loro competenza pragmatico-interazionale nell'elaborazione delle loro risposte: si tratta della presenza del ringraziamento (13 occorrenze sia in italiano che portoghese). Nella sequenza canonica di una richiesta il ringraziamento segue il turno dell'interlocutore che può soddisfare la richiesta o aderire alla necessità del richiedente, mostrandosi sensibile all'atto linguistico realizzato anche se non è in grado di fare quanto viene richiesto (Santoro, 2016). II fatto che in un DCT scritto compaia il ringraziamento fa quindi pensare a un'anticipazione in cui l'informatore salta il turno dell'interlocutore che viene presupposto per poter considerare conclusa la sequenza.

Tornando alla giustificazione, che come si diceva compare in una percentuale molto elevata di casi, vediamo che si delinea una chiara tendenza, che pare comune alle due lingue, 


\section{normas}

secondo la quale l'esposizione dei motivi è considerata un fattore che può rendere accettabile ed esaudibile la richiesta fatta.

Il riconoscimento del costo della richiesta in italiano è l'unico altro atto di supporto ad aver fatto registrare una percentuale che supera di qualche punto la soglia del 10\%: siamo al $15 \%$ e con questo risultato al terzo tipo di atto di supporto considerando la quantità di occorrenze. La percentuale in portoghese è un po' inferiore, ma comunque significativa, così come quella delle proposte di risarcimento o garanzia, un'altra delle strategie selezionate dai parlanti. Quasi del tutto assenti sono invece le proposte di alternative che, almeno nel campione studiato, non sono state incluse nella formulazione delle richieste.

Passiamo adesso ai modificatori e alla loro presenza nel corpus:

Tabella 4 - Modificatori nel corpus in italiano (IT) e portoghese brasiliano (PB)

\begin{tabular}{|c|c|c|c|}
\hline \multirow[b]{2}{*}{ MODIFICATORI MORFOSINTATTICI } & \multirow[b]{2}{*}{ Sigla } & \multicolumn{2}{|c|}{ Numero occorrenze } \\
\hline & & IT (240) & PB (240) \\
\hline Dislocamento verbale & $<\mathrm{mms} 1>$ & $\begin{array}{l}120 \\
(50 \%)\end{array}$ & $\begin{array}{l}99 \\
(41,25 \%)\end{array}$ \\
\hline Verbi modali e costruzioni equivalenti & $<\mathrm{mms} 2>$ & $\begin{array}{l}170 \\
(70,83 \%)\end{array}$ & $\begin{array}{l}122 \\
(50,93 \%)\end{array}$ \\
\hline Incassatura & $<\mathrm{mms3}>$ & $\begin{array}{l}1 \\
(0,41 \%)\end{array}$ & $\begin{array}{l}2 \\
(0,83 \%)\end{array}$ \\
\hline Negazione dell'interrogativa o formule dubitative & $<m m s 4>$ & $\begin{array}{l}14 \\
(5,83 \%)\end{array}$ & $\begin{array}{l}21 \\
(8,75 \%)\end{array}$ \\
\hline Diminutivi & $<m m s 5>$ & $\begin{array}{l}0 \\
(0 \%)\end{array}$ & $\begin{array}{l}8 \\
(3,33 \%)\end{array}$ \\
\hline \multicolumn{4}{|l|}{ MODIFICATORI LESSICALI } \\
\hline Minimizzatori o indeterminatori lessicali & $<\mathrm{m} \mid 1>$ & $\begin{array}{l}53 \\
(22,08 \%) \\
\end{array}$ & $\begin{array}{l}46 \\
(19,16 \%)\end{array}$ \\
\hline Dubitatori lessicali & $<m \mid 2>$ & $\begin{array}{l}19 \\
(7,91 \%)\end{array}$ & $\begin{array}{l}2 \\
(0,83 \%)\end{array}$ \\
\hline Rafforzatori lessicali & $<m \mid 3>$ & $\begin{array}{l}93 \\
(38,75 \%)\end{array}$ & $\begin{array}{l}115 \\
(47,91 \%)\end{array}$ \\
\hline Scelte lessicali attenuanti & $<m \mid 4>$ & $\begin{array}{l}19 \\
(7,91 \%)\end{array}$ & $\begin{array}{l}47 \\
(19,58 \%)\end{array}$ \\
\hline \multicolumn{4}{|l|}{ MODIFICATORI DISCORSIVI } \\
\hline Attivatori di vincolo o empatia & $<\mathrm{md1}>$ & $\begin{array}{l}2 \\
(0,83 \%)\end{array}$ & $\begin{array}{l}14 \\
(5,83 \%)\end{array}$ \\
\hline Marche di cortesia & $<m d 2>$ & $\begin{array}{l}63 \\
(26,25 \%)\end{array}$ & $\begin{array}{l}48 \\
(20 \%)\end{array}$ \\
\hline Riformulatori & $<m d 3>$ & $\begin{array}{l}2 \\
(0,83 \%) \\
\end{array}$ & $\begin{array}{l}0 \\
(0 \%)\end{array}$ \\
\hline
\end{tabular}

Spicca la predominanza di dislocamento verbale e verbi modali/costruzioni equivalenti che sono le strategie morfosintattiche più utilizzate ed evidentemente più "disponibili" in entrambe le lingue, spesso anche in combinazione. Se voglio chiedere qualcosa, tendo ad attenuare preferendo l'uso di tempi e modi verbali che trasmettano l'idea del distanziamento da quanto viene detto e dalla situazione reale, come a voler lasciare 


\section{normas}

Richieste e attenuazione | Elisabetta Santoro

maggiore spazio al destinatario della richiesta. L'uso dei verbi modali con un riferimento immediato alla modalità deontica e quindi all'asse che va dalla possibilità (dell'altro) alla necessità (dell'io) fa parte di una strategia per certi versi analoga perché si tende a lasciare all'altro libertà di scelta.

In ordine decrescente di presenza seguono i rafforzatori lessicali (93 in italiano, corrispondenti al 38,75\%, e 115 in portoghese, pari al 47,91\%), la cui presenza in entrambe le lingue ci fa riflettere sulla funzione che può avere questa strategia. Come si è già detto, la nostra supposizione è che si tratti di un modo per ridurre i rischi del parlante perché fa sembrare più giustificata la sua richiesta, ovvero, la sua "invasione" del territorio dell'altro.

Vanno citate anche le ritualizzate marche di cortesia che in italiano vengono utilizzate nel $26,25 \%$ dei casi e in portoghese nel 20\%. I diminutivi poco presenti anche in portoghese $(3,33 \%)$ sono del tutto assenti in italiano, mentre i riformulatori non compaiono mai in portoghese e solo 2 volte in tutto il corpus in italiano, indicando che si tratta in questo caso di un fenomeno dell'oralità che non si trasferisce nello scritto.

Passando ora alle variabili selezionate per verificare se e in che misura influiscono sull'uso di atti di supporto e modificatori, vediamo i risultati ottenuti interrogando il corpus dopo aver filtrato le richieste sulla base del grado di imposizione: 
Tabella 5 - Atti di supporto e grado di imposizione in italiano (IT) e portoghese brasiliano (PB)

\begin{tabular}{|c|c|c|c|c|c|}
\hline \multirow[t]{3}{*}{ Tipologia di atto di supporto } & \multirow[t]{3}{*}{ Sigla } & \multicolumn{4}{|c|}{$\begin{array}{l}\text { Numero occorrenze con grado di imposizione alto } \\
\text { (A) e basso (B) }\end{array}$} \\
\hline & & \multicolumn{2}{|l|}{$\mathrm{IT}$} & \multicolumn{2}{|l|}{ PB } \\
\hline & & $A(120)$ & $\mathrm{B}(120)$ & $A(120)$ & $\mathrm{B}(120)$ \\
\hline Appello & $<$ as1> & $\begin{array}{l}115 \\
(95,83 \%)\end{array}$ & $\begin{array}{l}64 \\
(53,33 \%)\end{array}$ & $\begin{array}{l}89 \\
(74,16 \%)\end{array}$ & $\begin{array}{l}58 \\
(48,33 \%)\end{array}$ \\
\hline Esclamazione & $<$ as2 $>$ & $\begin{array}{l}2 \\
(1,66 \%) \\
\end{array}$ & $\begin{array}{l}13 \\
(10,83 \%)\end{array}$ & $\begin{array}{l}5 \\
(4,16 \%) \\
\end{array}$ & $\begin{array}{l}15 \\
(1,25 \%)\end{array}$ \\
\hline Minimizzatore & $<$ as3 $>$ & $\begin{array}{l}19 \\
(15,83 \%)\end{array}$ & $\begin{array}{l}2 \\
(1,66 \%)\end{array}$ & $\begin{array}{l}12 \\
(1 \%)\end{array}$ & $\begin{array}{l}1 \\
(0,83 \%)\end{array}$ \\
\hline Preparatore & $<\mathrm{as} 4>$ & $\begin{array}{l}15 \\
(1,25 \%)\end{array}$ & $\begin{array}{l}2 \\
(1,66 \%)\end{array}$ & $\begin{array}{l}13 \\
(10,83 \%)\end{array}$ & $\begin{array}{l}0 \\
(0 \%)\end{array}$ \\
\hline Giustificazione & $<$ as5> & $\begin{array}{l}114 \\
(95 \%)\end{array}$ & $\begin{array}{l}57 \\
(47,5 \%)\end{array}$ & $\begin{array}{l}105 \\
(87,5 \%)\end{array}$ & $\begin{array}{l}47 \\
(39,16 \%)\end{array}$ \\
\hline Proposta di risarcimento o garanzia & $<$ as6> & $\begin{array}{ll}11 \\
(9,16 \%)\end{array}$ & $\begin{array}{l}8 \\
(6,66 \%)\end{array}$ & $\begin{array}{l}12 \\
(1 \%) \\
\end{array}$ & $\begin{array}{l}13 \\
(10,83 \%) \\
\end{array}$ \\
\hline Proposta di alternative & $<$ as7 $>$ & $\begin{array}{l}1 \\
(0,83 \%) \\
\end{array}$ & $\begin{array}{l}0 \\
(0 \%) \\
\end{array}$ & $\begin{array}{l}1 \\
(0,83 \%) \\
\end{array}$ & $\begin{array}{l}0 \\
(0 \%) \\
\end{array}$ \\
\hline Adulatore & $<$ as8 $>$ & $\begin{array}{l}15 \\
(1,25 \%)\end{array}$ & $\begin{array}{l}1 \\
(0,83 \%)\end{array}$ & $\begin{array}{l}3 \\
(2,5 \%)\end{array}$ & $\begin{array}{l}0 \\
(0 \%)\end{array}$ \\
\hline Ringraziamento & $<$ as9> & $\begin{array}{l}9 \\
(7,5 \%)\end{array}$ & $\begin{array}{l}4 \\
(3,33 \%) \\
\end{array}$ & $\begin{array}{ll}6 \\
(5 \%) \\
\end{array}$ & $\begin{array}{l}7 \\
(5,83 \%) \\
\end{array}$ \\
\hline $\begin{array}{l}\begin{array}{l}\text { Riconoscimento del costo della } \\
\text { richiesta }\end{array} \\
\end{array}$ & $<\operatorname{as} 10>$ & $\begin{array}{l}30 \\
(25 \%)\end{array}$ & $\begin{array}{l}6 \\
(5 \%)\end{array}$ & $\begin{array}{l}20 \\
(16,66 \%)\end{array}$ & $\begin{array}{l}3 \\
(2,5 \%)\end{array}$ \\
\hline
\end{tabular}

Come si vede, la variabile grado di imposizione si mostra altamente sensibile alla maggior parte degli atti di supporto selezionati e verificati nel corpus. Primi fra tutti colpiscono gli stessi due atti che già nel conteggio sul corpus completo si erano mostrati più produttivi, vale a dire: appello e giustificazione. In italiano la percentuale di appelli presenti nelle richieste "difficili", ovvero ad alto grado di imposizione, è del $42,5 \%$ superiore rispetto a quella delle richieste "facili"; anche in portoghese brasiliano la differenza è notevole, sebbene inferiore (25,83\%). Parrebbe dunque che chi ha risposto al questionario considera I'appello come una strategia che facilita I'approccio all'altro soprattutto quando quello che si sta per chiedere comporta un costo elevato.

Anche le occorrenze di giustificazioni equivalgono praticamente al doppio nelle richieste ad alto grado di imposizione, sia in italiano (114 / 57) che in portoghese (105 / 47), dimostrando che i parlanti hanno sentito molto più spesso la necessità di spiegare quando le loro richieste erano più costose.

Sebbene i numeri siano parecchio inferiori, un risultato dello stesso tipo è stato ottenuto anche con le occorrenze del riconoscimento del costo della richiesta e dei minimizzatori, concentrate quasi esclusivamente nelle richieste ad alto grado di imposizione. Una suddivisione analoga si osserva anche con gli adulatori che hanno, tuttavia, solo 3 occorrenze in portoghese, tutte concentrate nelle richieste ad alto grado di imposizione, e 
16 in italiano, di cui soltanto una compare quando il grado di imposizione è inferiore. L'ultima osservazione riguarda le esclamazioni stranamente quasi tutte concentrate, sia in italiano che in portoghese, nelle richieste a basso grado di imposizione.

Ci spostiamo adesso sui risultati ottenuti con la ricerca dei modificatori all'interno del corpus, sempre partendo dal filtro del grado di imposizione:

Tabella 6 - Modificatori e grado di imposizione in italiano (IT) e portoghese brasiliano (PB)

\begin{tabular}{|c|c|c|c|c|c|}
\hline \multirow{3}{*}{$\begin{array}{l}\text { MODIFICATORI } \\
\text { MORFOSINTATTICI }\end{array}$} & \multirow[t]{3}{*}{ Sigla } & \multicolumn{4}{|c|}{$\begin{array}{l}\text { Grado di imposizione } \\
\text { alto (A) /basso (B) }\end{array}$} \\
\hline & & \multicolumn{2}{|c|}{ IT } & \multicolumn{2}{|l|}{$\mathrm{PB}$} \\
\hline & & A (120) & $\mathrm{B}(120)$ & $\mathrm{A}(120)$ & $\mathrm{B}(120)$ \\
\hline Dislocamento verbale & $<\mathrm{mms1}>$ & $\begin{array}{l}77 \\
(64,16 \%)\end{array}$ & $\begin{array}{l}43 \\
(35,83 \%) \\
\end{array}$ & $\begin{array}{l}70 \\
(58,33 \%)\end{array}$ & $\begin{array}{l}29 \\
(24,16 \%) \\
\end{array}$ \\
\hline Verbi modali e costruzioni equivalenti & $<\mathrm{mms} 2>$ & $\begin{array}{l}111 \\
(92,5 \%)\end{array}$ & $\begin{array}{l}59 \\
(49,16 \%)\end{array}$ & $\begin{array}{l}77 \\
(64,16 \%)\end{array}$ & $\begin{array}{l}45 \\
(37,5 \%)\end{array}$ \\
\hline Incassatura & $<\mathrm{mms3}>$ & $\begin{array}{l}1 \\
(0,83 \%)\end{array}$ & $\begin{array}{l}0 \\
(0 \%)\end{array}$ & $\begin{array}{l}2 \\
(1,66 \%)\end{array}$ & $\begin{array}{l}0 \\
(0 \%)\end{array}$ \\
\hline $\begin{array}{l}\text { Negazione dell'interrogativa o formule } \\
\text { dubitative }\end{array}$ & $<\mathrm{mms} 4>$ & $\begin{array}{l}9 \\
(7,5 \%)\end{array}$ & $\begin{array}{l}5 \\
(4,16 \%)\end{array}$ & $\begin{array}{l}19 \\
(15,83 \%)\end{array}$ & $\begin{array}{l}2 \\
(1,66 \%)\end{array}$ \\
\hline Diminutivi & $<$ mms5 $>$ & $\begin{array}{l}0 \\
\\
(0 \%)\end{array}$ & $\begin{array}{l}0 \\
\\
(0 \%)\end{array}$ & 3 & $\begin{array}{l}5 \\
(4,16 \%)\end{array}$ \\
\hline \multicolumn{6}{|l|}{ MODIFICATORI LESSICALI } \\
\hline Minimizzatori o indeterminatori lessicali & $<\mathrm{m} \mid 1>$ & $\begin{array}{l}43 \\
(35,83 \%)\end{array}$ & $\begin{array}{l}10 \\
(8,33 \%)\end{array}$ & $\begin{array}{l}31 \\
(25,83 \%)\end{array}$ & $\begin{array}{l}15 \\
(1,25 \%)\end{array}$ \\
\hline Dubitatori lessicali & $<\mathrm{m} \mid 2>$ & $\begin{array}{l}4 \\
(3,33 \%)\end{array}$ & $\begin{array}{l}15 \\
(1,25 \%)\end{array}$ & $(0,83 \%)$ & $(0,83 \%)$ \\
\hline Rafforzatori lessicali & $<m \mid 3>$ & $\begin{array}{l}80 \\
(68,33 \%)\end{array}$ & $\begin{array}{l}13 \\
(10,83 \%)\end{array}$ & $\begin{array}{l}99 \\
(82,5 \%)\end{array}$ & $\begin{array}{l}16 \\
(13,33 \%)\end{array}$ \\
\hline Scelte lessicali attenuanti & $<m \mid 4>$ & $\begin{array}{l}17 \\
(14,16 \%)\end{array}$ & $\begin{array}{l}2 \\
(1,66 \%)\end{array}$ & $\begin{array}{l}29 \\
(24,16 \%)\end{array}$ & $\begin{array}{l}18 \\
(15 \%)\end{array}$ \\
\hline \multicolumn{6}{|l|}{ MODIFICATORI DISCORSIVI } \\
\hline Attivatori di vincolo o empatia & $<$ md1 $>$ & $\begin{array}{l}2 \\
(1,66 \%)\end{array}$ & $\begin{array}{l}0 \\
(0 \%) \\
\end{array}$ & $\begin{array}{l}4 \\
(3,33 \%)\end{array}$ & $\begin{array}{l}10 \\
(8,33 \%)\end{array}$ \\
\hline Marche di cortesia & $<m d 2>$ & $\begin{array}{l}32 \\
(26,66 \%)\end{array}$ & $\begin{array}{l}31 \\
(25,83 \%)\end{array}$ & $\begin{array}{l}25 \\
(20,83 \%)\end{array}$ & $\begin{array}{l}23 \\
(19,16 \%)\end{array}$ \\
\hline Riformulatori & $<m d 3>$ & $\begin{array}{l}1 \\
(0,83 \%)\end{array}$ & $\begin{array}{l}1 \\
(0,83 \%)\end{array}$ & $\begin{array}{l}0 \\
0 \%)\end{array}$ & $\begin{array}{l}0 \\
(0 \%)\end{array}$ \\
\hline
\end{tabular}

Anche i modificatori si sono rivelati fortemente sensibili alla variabile e i più frequenti nel corpus, vale a dire dislocamento verbale e verbi modali/costruzioni equivalenti, si concentrano nelle richieste ad alto grado di imposizione, ovvero quelle considerate più difficili. Confrontando le percentuali si constata in italiano una differenza pari al 28,33\% nel caso del dislocamento verbale e del $43,34 \%$ per i verbi modali/costruzioni equivalenti; in portoghese, invece, il primo tipo di modificatore compare con una differenza percentuale pari al 34,17 e il secondo al 26,66\%. 


\section{normas}

L'altra strategia che merita attenzione è quella dei rafforzatori lessicali che si concentrano nelle richieste ad alto grado di imposizione con un numero di occorrenze che non lascia dubbi sull'assoluta predominanza del loro uso in condizioni di maggiore disagio per chi chiede. In italiano abbiamo ben 80 occorrenze nelle richieste "difficili" contro 13 in quelle "facili" e in portoghese la relazione è di 99 a 16. I minimizzatori o indeterminatori lessicali e, pur se in numero inferiore, le scelte lessicali attenuanti si suddividono seguendo lo stesso standard. In generale, si può affermare che nel nostro campione ad un livello di imposizione più elevato corrisponde senza dubbio una quantità di strategie attenuative notevolmente superiore e quindi un'evidente preoccupazione riguardo a ciò che viene detto che si traduce in procedimenti linguistici che tendono a creare un equilibrio e a mitigare. Colpisce però che le marche di cortesia siano presenti in numero quasi identico nei due tipi di richiesta così da non sembrare sensibili al grado di imposizione. È probabilmente il loro uso ritualizzato e quasi automatico a causare questo effetto.

La seconda variabile indipendente che, secondo le nostre ipotesi iniziali, avrebbe potuto influenzare l'uso di strategie attenuative è la distanza sociale. La tabella che segue contiene i risultati relativi agli atti di supporto in combinazione con questa variabile:

Tabella 7 - Atti di supporto e distanza sociale in italiano (IT) e portoghese brasiliano (PB)

\begin{tabular}{|c|c|c|c|c|c|}
\hline \multirow[t]{3}{*}{ Tipologia di atto di supporto } & \multirow[t]{3}{*}{ Sigla } & \multicolumn{4}{|c|}{$\begin{array}{l}\text { Numero occorrenze con distanza sociale } \\
\text { alta (A) e bassa (B) }\end{array}$} \\
\hline & & \multicolumn{2}{|l|}{ IT } & \multicolumn{2}{|l|}{$\mathrm{PB}$} \\
\hline & & $A(120)$ & $\mathrm{B}(120)$ & $A(120)$ & $\mathrm{B}(120)$ \\
\hline Appello & $<$ as1 $>$ & $\begin{array}{l}60 \\
(50 \%)\end{array}$ & $\begin{array}{l}119 \\
(99,16 \%)\end{array}$ & $\begin{array}{l}55 \\
(45,83 \%)\end{array}$ & $\begin{array}{l}92 \\
(76,66 \%)\end{array}$ \\
\hline Esclamazione & $<$ as2> & $\begin{array}{l}14 \\
(11,66 \%)\end{array}$ & $\begin{array}{l}1 \\
(0,83 \%)\end{array}$ & $\begin{array}{l}15 \\
(12,5 \%)\end{array}$ & $\begin{array}{l}5 \\
(4,16 \%)\end{array}$ \\
\hline Minimizzatore & $<a s 3>$ & 8 & $\begin{array}{l}13 \\
(10,83 \%)\end{array}$ & 4 & $\begin{array}{l}9 \\
(7,5 \%)\end{array}$ \\
\hline Preparatore & $<\mathrm{as} 4>$ & $\begin{array}{l}5 \\
(4,16 \%)\end{array}$ & $\begin{array}{l}12 \\
(1 \%)\end{array}$ & 3 & $\begin{array}{l}10 \\
(8,33 \%)\end{array}$ \\
\hline Giustificazione & $<$ as5 $>$ & $\begin{array}{l}95 \\
(79,16 \%)\end{array}$ & \begin{tabular}{|l|}
76 \\
$(63,33 \%)$
\end{tabular} & $\begin{array}{l}75 \\
(62,5 \%)\end{array}$ & $\begin{array}{l}77 \\
(64,16 \%)\end{array}$ \\
\hline Proposta di risarcimento o garanzia & $<a s 6>$ & $\begin{array}{l}11 \\
(9,16 \%)\end{array}$ & $\begin{array}{l}8 \\
(6,66 \%)\end{array}$ & $\begin{array}{l}15 \\
(12,5 \%)\end{array}$ & $\begin{array}{l}10 \\
(8,33 \%)\end{array}$ \\
\hline Proposta di alternative & $<$ as7> & $\begin{array}{l}1 \\
(0,83 \%)\end{array}$ & $\begin{array}{l}0 \\
10 \%)\end{array}$ & $\begin{array}{l}0 \\
0 \%)\end{array}$ & $\begin{array}{l}1 \\
(0,83 \%)\end{array}$ \\
\hline Adulatore & $<$ as8 $>$ & 9 & 7 & 1 & 2 \\
\hline Ringraziamento & $<$ as9> & $\begin{array}{l}5 \\
(4,16 \%)\end{array}$ & $\begin{array}{l}8 \\
(6,66 \%)\end{array}$ & $\begin{array}{l}4 \\
(3,33 \%)\end{array}$ & $\begin{array}{l}9 \\
(7,5 \%)\end{array}$ \\
\hline $\begin{array}{l}\text { Riconoscimento del costo della } \\
\text { richiesta }\end{array}$ & $<$ as10> & $\begin{array}{l}16 \\
(13,33 \%)\end{array}$ & $\begin{array}{l}20 \\
(16,66 \%)\end{array}$ & $\begin{array}{l}9 \\
(7,5 \%)\end{array}$ & $\begin{array}{l}14 \\
(11,66 \%)\end{array}$ \\
\hline
\end{tabular}

Il primo aspetto che colpisce chi osserva i risultati è che in questo caso le occorrenze di appello sono concentrate nelle richieste a bassa distanza sociale, quando cioè la relazione tra gli interlocutori è di "vicinanza". In italiano praticamente tutte le richieste formulate in questo tipo di situazione (119 su 120) contengono un appello. II numero si riduce alla metà (60 su 120), quando la distanza sociale è alta. In portoghese la tendenza è simile, ma il numero di occorrenze inferiore (92 su 120 e 55 su 120). 
Le giustificazioni delle richieste variano in misura molto inferiore rispetto a quanto era avvenuto per la variabile grado di imposizione, dimostrando che si giustificano le richieste sia con chi si conosce bene che con chi si conosce poco o per niente. In italiano lo scarto tra la distanza sociale bassa e alta è superiore rispetto al portoghese con 95 occorrenze nel caso di interlocutori distanti e 76 tra parlanti più vicini tra loro, mentre in portoghese il numero è praticamente identico (75 / 77).

Negli altri tipi di strategia attenuativa si osserva una tendenza a una maggiore presenza quando la distanza tra gli interlocutori è alta, ma con differenze quantitativamente meno marcate.

Le esclamazioni, che avevamo visto comparire quasi esclusivamente nelle richieste a basso grado di imposizione, sono in questa suddivisione concentrate nei casi di alta distanza sociale.

Nell'ultima tabella riportiamo i risultati ottenuti interrogando il corpus filtrato ancora una volta sulla base della variabile distanza sociale, ma questa volta per verificare se e in quale misura fossero presenti i modificatori selezionati:

Tabella 8 - Modificatori e distanza sociale in italiano (IT) e portoghese brasiliano (PB)

\begin{tabular}{|c|c|c|c|c|c|}
\hline \multirow{3}{*}{ MODIFICATORI MORFOSINTATTICI } & \multirow[t]{3}{*}{ Sigla } & \multicolumn{2}{|l|}{ IT } & \multicolumn{2}{|l|}{ PB } \\
\hline & & \multicolumn{4}{|c|}{\begin{tabular}{|l|} 
Distanza sociale \\
alta $(\mathrm{A})$ e bassa $(\mathrm{B})$ \\
\end{tabular}} \\
\hline & & A & $\mathrm{B}$ & A & $\mathrm{B}$ \\
\hline Dislocamento verbale & $<\mathrm{mms} 1>$ & $\begin{array}{l}45 \\
(37,5 \%)\end{array}$ & $\begin{array}{l}75 \\
(62,5 \%)\end{array}$ & $\begin{array}{l}60 \\
(50 \%)\end{array}$ & $\begin{array}{l}39 \\
(32,5 \%)\end{array}$ \\
\hline Verbi modali e costruzioni equivalenti & $<m m s 2>$ & $\begin{array}{l}76 \\
(63,33 \%)\end{array}$ & $\begin{array}{l}94 \\
(78,33 \%)\end{array}$ & $\begin{array}{l}63 \\
(52,5 \%) \\
\end{array}$ & $\begin{array}{l}59 \\
(49,16 \%) \\
\end{array}$ \\
\hline Incassatura & $<\mathrm{mms} 3>$ & $\begin{array}{l}1 \\
(0,83 \%)\end{array}$ & $\begin{array}{l}0 \\
(0 \%)\end{array}$ & $\begin{array}{l}2 \\
(1,66 \%)\end{array}$ & $\begin{array}{l}0 \\
(0 \%)\end{array}$ \\
\hline $\begin{array}{l}\text { Negazione dell'interrogativa } 0 \text { formule } \\
\text { dubitative }\end{array}$ & $<m m s 4>$ & $\begin{array}{l}8 \\
(6,66 \%)\end{array}$ & $\begin{array}{l}6 \\
(5 \%)\end{array}$ & $\begin{array}{l}8 \\
(6,66 \%)\end{array}$ & $\begin{array}{l}13 \\
(10,83 \%)\end{array}$ \\
\hline Diminutivi & $<$ mms5 $>$ & $\begin{array}{l}0 \\
(0 \%) \\
\end{array}$ & $\begin{array}{l}0 \\
(0 \%) \\
\end{array}$ & $\begin{array}{l}8 \\
(6,66 \%) \\
\end{array}$ & $\begin{array}{l}0 \\
(0 \%) \\
\end{array}$ \\
\hline \multicolumn{6}{|l|}{ MODIFICATORI LESSICALI } \\
\hline Minimizzatori o indeterminatori lessicali & $<\mathrm{m} \mid 1>$ & $\begin{array}{l}14 \\
(11,66 \%)\end{array}$ & $\begin{array}{l}39 \\
(32,5 \%)\end{array}$ & $\begin{array}{l}1 \\
(25,83 \%)\end{array}$ & $\begin{array}{l}15 \\
(12,5 \%)\end{array}$ \\
\hline Dubitatori lessicali & $<\mathrm{m} \mid 2>$ & $\begin{array}{l}3 \\
(2,5 \%)\end{array}$ & $\begin{array}{l}16 \\
(13,33 \%)\end{array}$ & $\begin{array}{l}2 \\
(1,66 \%)\end{array}$ & $\begin{array}{l}0 \\
(0 \%)\end{array}$ \\
\hline Rafforzatori lessicali & $<m \mid 3>$ & $\begin{array}{l}45 \\
(37,5 \%)\end{array}$ & $\begin{array}{l}48 \\
(40 \%)\end{array}$ & $\begin{array}{l}63 \\
(52,5 \%)\end{array}$ & $\begin{array}{l}52 \\
(43,33 \%)\end{array}$ \\
\hline Scelte lessicali attenuanti & $<m \mid 4>$ & $\begin{array}{l}8 \\
(6,66 \%) \\
\end{array}$ & $\begin{array}{l}11 \\
(9,16 \%) \\
\end{array}$ & $\begin{array}{l}29 \\
(24,16 \%)\end{array}$ & $\begin{array}{l}18 \\
(15 \%)\end{array}$ \\
\hline \multicolumn{6}{|l|}{ MODIFICATORI DISCORSIVI } \\
\hline Attivatori di vincolo o empatia & $<\mathrm{md1}>$ & $\begin{array}{l}0 \\
(0 \%)\end{array}$ & $\begin{array}{l}2 \\
(1,66 \%)\end{array}$ & $\begin{array}{l}6 \\
(5 \%)\end{array}$ & $\begin{array}{l}8 \\
(6,66 \%)\end{array}$ \\
\hline Marche di cortesia & $<m d 2>$ & $\begin{array}{l}28 \\
(23,33 \%)\end{array}$ & $\begin{array}{l}35 \\
(29,16 \%)\end{array}$ & $\begin{array}{l}31 \\
(25,83 \%)\end{array}$ & $\begin{array}{l}17 \\
(14,16 \%)\end{array}$ \\
\hline Riformulatori & $<m d 3>$ & $\begin{array}{l}0 \\
10 \%)\end{array}$ & $\begin{array}{l}2 \\
(1,66 \%)\end{array}$ & $\begin{array}{l}0 \\
(0 \%)\end{array}$ & $\begin{array}{l}0 \\
10 \%)\end{array}$ \\
\hline
\end{tabular}




\section{normas}

Le tendenze che si osservano in questo caso sono decisamente meno univoche e, talvolta, più difficili da interpretare, giacché sembrano rivelare una diversa sensibilità in italiano e in portoghese brasiliano rispetto alla variabile presa in esame. Dislocamento verbale e verbi modali/costruzioni equivalenti che, come si è visto, rappresentano le strategie di modificazione con maggiore presenza nel corpus, si suddividono in modo non lineare e molto meno marcato rispetto a quanto avviene analizzando la variabile grado di imposizione. In italiano il dislocamento verbale compare 75 volte $(62,5 \%)$ nelle richieste a bassa distanza sociale e $45(37,5 \%)$ in quelle tra interlocutori più distanti tra loro; in portoghese, invece, sono presenti 60 occorrenze (50\%) nel primo caso e solo 39 (32,5\%) nel secondo. Verbi modali e costruzioni equivalenti sono stati utilizzati un maggior numero di volte quando i parlanti nativi di italiano hanno immaginato un interlocutore "vicino" (94 occorrenze corrispondenti al $78,33 \%$ del totale di richieste) rispetto alle situazioni in cui I'interlocutore era distante (76 occorrenze che equivalgono al 63,33\%). Per i parlanti nativi di portoghese brasiliano la variabile non ha prodotto variazioni significative su questa strategia (in un caso le occorrenze sono 59 e nell'altro 63, con una differenza pari al 3,34\%).

Anche l'uso dei rafforzatori lessicali non si è mostrato molto sensibile alla variabile selezionata. Nei parlanti brasiliani si osserva tuttavia un uso quantitativamente superiore di minimizzatori o indeterminatori lessicali, scelte lessicali attenuanti e diminutivi nelle situazioni di più elevata distanza sociale.

Un'ultima osservazione sul risultato relativo alle marche di cortesia in relazione alla distanza sociale: in italiano sono in numero poco superiore nelle situazioni considerate di bassa distanza sociale, ovvero tra amici o parenti, ma la relazione si inverte in portoghese, considerato che nelle situazioni ad alta distanza sociale la percentuale è superiore rispetto a quelle a bassa distanza sociale (31 a 17 con una differenza percentuale pari all'11,67\%), contraddicendo in parte quanto si era osservato nelle richieste che variavano in base al grado di imposizione, dove il numero di occorrenze era in tutti i casi pressoché identico e facendo pensare che, almeno in portoghese, il tipo di rapporto con l'interlocutore produce un effetto sulla scelta di usare questa strategia che invece non viene causato dal tipo di richiesta.

\section{ALCUNE RIFLESSIONI CONCLUSIVE}

Lo studio realizzato ha permesso di verificare analogie e differenze nelle richieste di parlanti nativi di italiano e di portoghese brasiliano, oltre che di identificare ricorrenze e tendenze che potranno essere usate anche per ricerche future. Si è visto che nel campione analizzato gli atti di supporto più produttivi, sebbene in italiano si presentino in numero maggiore, sono l'appello [179 (IT) - 147 (PB)] e la giustificazione [171 (IT) - 152 (PB)]. Pur avendo scritto e quindi dovuto immaginare che cosa avrebbero detto nelle situazioni indicate, i parlanti nativi di entrambe le lingue hanno dimostrato di considerare adeguato stabilire un contatto con l'interlocutore prima di passare alla richiesta vera e propria. Si tratta di un tipo di mossa attenuativa che fa leva sull'empatia e che tende a captare la "benevolenza" di chi viene prescelto come interlocutore. Ė, d'altro canto, significativo che il secondo posto sia occupato dalle giustificazioni, molte volte presenti anche nelle richieste considerate "facili": "non posso chiedere qualcosa senza spiegare perché la chiedo" sembra essere il messaggio trasmesso dalla marcata presenza di questo atto di supporto. 


\section{normas}

In generale, è stato utile osservare le strategie che si possono considerare caratteristiche dell'interazione. Di fatto, nonostante non si sia prevista una risposta, ovvero un turno dell'interlocutore, sono presenti elementi che segnalano la "riproduzione", anche nello scritto e in una situazione monologica, di aspetti tipici dell'interazione come il ringraziamento, che comparirebbe normalmente solo in un turno successivo, ma anche l'appello o le esclamazioni.

Per quanto concerne i modificatori, osserviamo riassumendo che anche in questo caso esiste un'analogia tra italiano e portoghese brasiliano, giacché, per quanto con una presenza non identica, i più produttivi sono stati in entrambe le lingue verbi modali $e$ costruzioni equivalenti [170 (IT) - 122 (PB)], dislocamento verbale [120 (IT) - 99 (PB)] e rafforzatori lessicali [93 (IT) - 115 (PB)]. Soprattutto questi ultimi, nonostante il loro nome, hanno, dal nostro punto di vista, un valore che riduce la forza illocutoria della richiesta perché, sottolineandone l'assoluta necessità, diminuisce il campo d'azione del richiedente, ma aumenta la sua possibilità di assicurarsi la comprensione e la buona disposizione dell'interlocutore.

A proposito del grado di imposizione, si è constatato che gli atti di supporto si sono rivelati molto sensibili a questa variabile, in particolare gli appelli e le giustificazioni, con un numero di occorrenze molto più elevato quando il grado di imposizione è alto. Anche i modificatori si mostrano sensibili alla variabile: spiccano soprattutto verbi modali e costruzioni equivalenti, dislocamento verbale, rafforzatori e minimizzatori. È interessante osservare, al contrario, l'equilibrio nel numero delle marche di cortesia, il cui uso pare indifferente al costo della richiesta, come se la sua ritualizzazione lo rendesse "automatico" a prescindere dal costo di ciò che si chiede.

Anche quando la variabile isolata è la distanza sociale, gli atti di supporto più sensibili sono, ancora una volta, appelli e giustificazioni. L'appello ha un numero di occorrenze molto più elevato nel caso in cui la distanza sociale è bassa sia in italiano $(B=119-A=60)$ che in portoghese brasiliano $(B=92-A=55)$, dato questo che parrebbe evidenziare che nelle situazioni di maggiore familiarità i parlanti tendono a utilizzare forme di ulteriore avvicinamento. Nei rapporti caratterizzati da una distanza sociale alta le giustificazioni sono in numero più elevato in italiano $(B=95-A=76)$, ma non in portoghese brasiliano $(B=75$ - A = 77), dove la loro presenza rimane pressoché identica. Per quanto riguarda i modificatori, il comportamento osservato nelle due lingue è spesso inverso, evidenziando la necessità di approfondimenti e di osservazioni più precise sul modo in cui si riflette sull'uso di questa strategia linguistica la distanza sociale o grado di familiarità.

A partire da questa prima analisi, si aprono diverse prospettive, tra cui: approfondire l'analisi anche qualitativa delle categorie e delle variabili che si sono mostrate più sensibili o che hanno rivelato le principali analogie e differenze, con una particolare attenzione alla posizione in cui compaiono i mezzi linguistici identificati e alla loro funzione specifica; applicare il modello di questo campione a tutto il corpus (il target è di 500 informatori per ogni lingua); incrociare i risultati ottenuti con altre variabili tra cui, ad esempio, età e genere; confrontare questi risultati con quelli ottenuti tramite altre metodologie di raccolta dei dati, andando via via da quelle più controllate a quelle meno controllate. Sarà interessante osservare anche se e in che misura compare un atto principale e se si tratta di un atto diretto o indiretto, cosa che potrà evidenziare altre caratteristiche del modo in cui le richieste si collegano all'attenuazione. 


\section{RIFERIMENTI BIBLIOGRAFICI}

BLUM-KULKA, Shoshana \& OLSHTAIN, Elite (1984): «Requests and Apologies: A Cross-Cultural Study of Speech Act Realization Patterns (CCSARP)", Applied Linguistics 5, 1984, 196-213, https://doi.org/10.1093/applin/5.3.196.

BLUM-KULKA, Shoshana, HouSE, Juliane \& KASPER, Gabriele, eds. (1989): Requests and Apologies: A CrossCultural Study of Speech Act Realization Patterns (CCSARP), Norwood, Ablex.

BRIZ, Antonio \& ALBELDA, Marta (2013): «Una propuesta teórica y metodológica para el análisis de la atenuación lingüística en español y portugués. La base de un proyecto común (ES.POR.ATENUACIÓN)», Onomazéin, 28, 288-319, https://doi.org/10.7764/onomazein.28.21.

Brown, Penelope \& LeVInson, Stephen C. (1987): Politeness. Some universals in language usage, Cambridge, Cambridge University Press.

CAFFI, Claudia (1999): «On mitigation», Journal of Pragmatics 31, 881-909, http://dx.doi.org/10.1016/S03782166(98)00098-8.

CAFFI, Claudia (2001): La mitigazione: un approccio pragmatico alla comunicazione nei contesti terapeutici, Münster, Hamburg, London: LIT.

CAFFI, Claudia (2007): Mitigation, London, Elsevier.

FAERCH, Claus \& KASPER, Gabriele (1989): «Internal and external modification in interlanguage request realization", in S. Blum-Kulka, J. House \& G. Kasper, eds.: Requests and Apologies: A Cross-Cultural Study of Speech Act Realization Patterns (CCSARP), Norwood, Ablex.

FÉLIX-BRASDEFER, J. César (2010): «Data collection methods in speech act performance: DCTs, role plays, and verbal reports», in A. Martínez-Flor \& E. Usó-Juan (eds.), Speech act performance: Theoretical, empirical and methodological issues, Amsterdam-Philadelphia, John Benjamins, 41-56.

FRASER, Bruce (1980): "Conversational mitigation», Journal of Pragmatics 4, 341-350, http://dx.doi.org/10.1016/0378-2166(80)90029-6.

GARCía Carmen, Apologizing in English: Politeness strategies used by native and non-native speakers, in Multilingua, 8, 1989, 3-20.

GoFFMAN, Erving (1967): Interaction ritual. Essays on face-to-face behaviour, New York, Pantheon Books (trad. it. Modelli di interazione, Bologna, il Mulino, 1971).

HAVERKATE, Henk (1994): La cortesía verbal. Estudio pragmalingüístico, Madrid, Gredos.

HYMES, Dell (1962): «The ethnography of speaking», in T. Galdwin \& W. C. Sturtevant, eds.: Anthropology and human behavior, Washington, DC, Anthropological Society of Washington, 13-53.

HYMES, Dell (1972): «On communicative competence», in J.B. Pride \& J. Holmes, eds.: Sociolinguistics, London, Penguin, 269-293.

HYMES, Dell (1974): «Ways of speaking», in R. Bauman \& J. Sherzer, eds.: Explorations in the ethnography of speaking, Cambridge, Cambridge University Press, 433-452.

MACKEY, Alison \& GASS, Susan M. (2005): Second Language Research. Methodology and Design, Malwah, New Jersey, Lawrence Erlbaum Associates.

MARIOTTINI, Laura (2007): La cortesia, Roma, Carocci.

Nuzzo, Elena (2007): Imparare a fare cose con le parole. Richieste, proteste, scuse in italiano lingua seconda, Perugia, Guerra Edizioni.

PALlotTI, Gabriele (2001): "L'ecologia del linguaggio: contestualizzazione dei dati e costruzione di teorie», in F. Albano Leoni, E. Stenta Krosbakken, R. Sornicola, C. Stromboli, C., a cura di: Dati empirici e teorie linguistiche. Atti del XXXIII Congresso Internazionale di Studi della Società di Linguistica Italiana, Roma, Bulzoni, 37-57.

SANTORO, Elisabetta (2012): «A constituição de um corpus de italiano falado para o estudo de pedidos e pedidos de desculpas: considerações sobre a validade interna e externa dos dados", in H. Mello, M. Pettorino, T. Raso, eds.: Proceedings of the VIIth GSCP International Conference: Speech and Corpora. ISBN 978-886655-351-9 (online), Firenze, Firenze University Press.

SANTORO, Elisabetta (2013): «Lo sviluppo della competenza pragmatica in italiano L2: un esperimento a partire da un corso online», in M. Rückl, E. Santoro \& I. Vedder, a cura di: Contesti di apprendimento in italiano L2. Tra teoria e pratica didattica, Firenze, Franco Cesati Editore, 27-42.

SANTORO, Elisabetta (2016): «lllocuzione e interazione nelle richieste in italiano: un confronto tra parlanti nativi e apprendenti brasiliani ", in E. Santoro \& I. Vedder, a cura di: Pragmatica e interculturalità in italiano lingua seconda, Firenze, Franco Cesati Editore, 41-52.

SBISÀ, Marina (2001): «lllocutionary force and degrees of strength in language use», Journal of Pragmatics 33, 1791-1814, http://dx. doi.org/10.1016/S0378-2166(00)00060-6.

THOMAS, Jenny (1983): «Cross-Cultural Pragmatic Failure», Applied Linguistics, 4, 91-112, https://doi.org/10.1093/applin/4.2.91. 
SIGLE UTILIZZATE

IT = italiano

$\mathrm{PB}=$ portoghese brasiliano

$\mathrm{A}=$ alto/alta (per grado di imposizione e distanza sociale)

$\mathrm{B}=$ basso/bassa (per grado di imposizione e distanza sociale) 